\title{
Limb Body Wall Complex
}

\author{
Surg Cdr J D'Souza*, Surg Cdr IK Indrajit ${ }^{+}$, Surg Cdr S Menon ${ }^{\#}$
}

MJAFI 2004; 60 : 77-80

Key Words : Abdominal wall defects; Limb-body-wall complex; Ultrasonography

\section{Introduction}

Timb-body wall complex (LBWC) is a rare, complicated, polymalformative fetal malformation syndrome with the essential features of : a) exencephaly/ encephalocele with facial clefts, b) thoraco-and/or abdominoschisis and c) limb defect [1]. Generally, the diagnosis is based on two of three of the above features. Two phenotypes have been described, the "placentocranial" and "placento-abdominal" adhesion phenotypes. This complex malformation, has no sex or familial predilection and is invariably fatal. The poor prognosis of LBWC necessitates an early antenatal diagnosis. Serum alpha-fetoprotein measurement and ultrasonographic examination is the key to prenatal diagnosis. The sonographic hallmark of LBWC is characterized by thoraco- and/or abdominoschisis, neural-tube abnormalities, severe scoliosis, positional deformities and abnormalities of fetal membranes [2]. In this case report we highlight the sonographic and colour flow imaging findings in LBWC presenting on routine antenatal scanning, along with the plain radiographs and the photographs of the aborted fetus.

\section{Case Report}

A 31 year old wife of a serving sailor presented to this hospital for routine antenatal ultrasonography scanning on 29 Sep 01. She was G1 P0 A0, at the time of scanning. She gave a history of a non-consanguineous marriage, did not take any drugs or chronic medication and was a booked case.

Ultrasound scans revealed a male fetus with the derived gestation age as 18 weeks 5 days (using her last menstrual period on 21 Jun 2001) and biparietal diameter as 16 weeks 2 days. The scans also revealed a large ill-defined abdominal wall defect through which the abdominal contents herniated into the extra embryonic coelom. The eviscerated organs formed a complex, bizarre appearing mass entangled with membranes (Fig 1). The diaphragm was absent. There was evidence to suggest bowel atresia, renal agenesis, anal atresia and lack of external genitalia. There was a lack of developed pelvic organs and perineum. There was evidence of scoliosis. While the fetal head diameter was normal for gestational age, the thoracic and abdominal diameters were disproportionately reduced. No anomalies were seen at the fetal eyes, facial profile, palate, lips, neck, or fetal cardiac and lung structures. Placenta was fundal and right lateral in position, showing grade 1 changes. Colour flow imaging showed evidence of a single umbilical artery within a short umbilical cord connecting the fetus (Fig 2). Colour flow imaging also clearly delineated the abnormal fetoplacental attachment. A provisional diagnosis of LBWC was made, based on the above sonographic findings.

The patient was informed of the poor prognosis and, after counselling, the patient elected to terminate the pregnancy. She was admitted as a high-risk pregnancy on 1 October 2001. The pregnancy was terminated with mesoprostol in lignocaine (400 mg), administered intravaginally in three doses five hours apart. Subsequently on 2 October 2001, she aborted a male fetus completely along with placenta and membranes.

The diagnosis of LBWC was confirmed after delivery. The severity of the defects and a distorted appearance made recognition of the lower half of the fetus difficult. The detailed examination of the fetus (Fig $3 \mathrm{~A}$ and $\mathrm{B}$ ) revealed herniation of abdominal contents through a large defect. The abdominal circumference was markedly diminished. The eviscerated organs formed a complex, bizarre appearing mass entangled with membranes. An extracorporeal liver was present. The right lower limb was abnormally hyperflexed at hip joint and clubfoot was present at right foot. There was evidence of marked scoliosis. The umbilical cord was short, straight, incompletely covered by amnion and adherent to the placental membranes as well as the eviscerated mass. The umbilical vessels were embedded in an amniotic sheet, which connected the skin margin of the anterior body wall defect to the placenta. The cut section of the umbilical cord revealed a single umbilical artery. Plain radiographs of the aborted fetus (Fig 4A and B) revealed evidence of marked scoliosis. The thoracic circumference was markedly diminished. After delivery she was advised that there was no familial predilection or no known recurrence risk. After counselling to report for regular screening in her future pregnancies to the hospital, she was discharged.

\section{Discussion}

LBWC is a rare complicated, polymalformative fetal malformation syndrome with the essential features of : a) exencephaly/encephalocele with facial clefts, b) thoraco- and/or abdominoschisis, and c) limb defect. Generally the diagnosis is based on two or three of the above features [1]. Coelosomia is found in all cases, it variably coexists with encephalic, vertebral, visceral or limb anomalies. This congenital anomaly has no sex or

\footnotetext{
${ }^{*+}$ Classified Specialist (Radiodiagnosis and Imaging), ${ }^{\#}$ Medical Officer, INHS Asvini, Colaba, Mumbai.
} 
familial predilection or known recurrence risk. Karyotype study has been reported to be normal in all cases of LBWC. LBWC is invariably fatal $[2,3,4,5]$. The incidence of this uncommon disorder is 1 in 4000 .

The pathogenesis of LBWC is unclear and uncertain. Three pathogenetic mechanisms have been proposed namely, the early amnion rupture theory, vascular disruption theory and embryonic dysgenesis. Similarly, Russo et al have proposed that there are two clearly distinguishable phenotypes : the placento-cranial" and "placento-abdominal" adhesion phenotype [6,7]. The first phenotype shows craniofacial defects and amniotic bands and/or adhesion, while the second is without craniofacial defects and presents urogenital anomalies, anal atresia, abdominal placental attachment, together with a persistence of the extra-embryonic celom. In addition, the authors attribute the two phenotypes as consequences of different pathogenetic mechanisms : firs type related to an early vascular disruption, while the second type is attributable to an intrinsic embryonal maldevelopment. While the first two theories can explain the form with "placento-cranial" adhesion, the "defective folding process" allows for a better explanation of the form with "palacento-cranial" adhesion. Also, Smith et al have suggested that LBWC results from amnion rupture caused by vascular or mechanical compression occurring between third and fifth weeks $[3,4]$. In our case report, the pattern of presentation resembled the "placento-abdominal" adhesion phenotype.

Some consider that LBWC simply represents a severe form of amniotic band syndrome. This is reinforced by the presence of amniotic bands in nearly $40 \%$ cases of LBWC. Moreover, some of the limb defects can be explained by the presence of amniotic bands. More recently, Van Allen [4] suggests that the presence of amniotic bands is a consequence of early vascular disruption, but is not the primary abnormality itself. Allen, in a study of a series of fetuses with LBWC found that the major structural defects included limb defects $(95 \%)$; marked scoliosis $(77 \%)$, internal organ malformation (95\%), craniofacial defects $(56 \%)$ and limb defects including club foot $(32 \%)$. The internal organ malformation present in $95 \%$ cases was cardiac defects, absent diaphragm, bowel atresia, renal agenesis and renal dysplasia. Body defects was a central feature of LBWC. Similarly, Patten et al reported trunk defects in $92 \%$ of the 13 fetuses, which exhibited features of LBWC [2]. Another large series by Van Allen et al found body defects in $96 \%$ of cases. The involvement of both abdomen and thorax was a more common feature as compared to involvement of either abdomen or thorax solely. The abdominal and thoracic contents herniate through a large defect into the extra embryonic celom. Typically, the eviscerated organs form a complex, bizarre appearing mass entangled with membranes. In our case the body defect was limited to the abdomen, even though the diaphragm was not identified.

Van Allen et al have divided the limb defects into 3 pathogenetic groups : a) secondary to disruption of embryonic vessels and surrounding tissue $(84 \%), b)$ secondary to amniotic bands or adhesions (16\%), and c) deformation versus haemorrhage ( $44 \%$ with club feet), with some fetuses having more than one pathogenetic mechanism causing limb defects, based on a study of limb defects from 25 fetuses with LBWC [6]. Majority of limb defects were hypothesized to have resulted from disruption of embryonic vessels. Importantly, in the specimens with amniotic band related limb defects, the most likely pathogenesis was identified as mechanical rupture through the amnion in the presence of a persistent extraembryonic celom or from adhesion of the amnion to necrotic embryonic tissue after the initial disruptive event. Clubfeet were present in $44 \%$ and was due either to disruption of embryonic vessels or to deformation.

The poor prognosis of LBWC necessitates an early antenatal diagnosis. Ultrasonographic detection of abdominoschisis, scoliosis, abnormalities of the lower extremities, a single umbilical artery, a short umbilical cord and an extremely elevated level of MSAFP is the key to early diagnosis. Ultrasonographically, the principal findings are the thoracoabdominal defect, limb anomalies, spinal and cord abnormalities [6]. All these groups of defects were present in our case. The severity of the defects and a distorted appearance of the fetus make recognition of the fetal parts difficult in almost all cases of LBWC. Spinal abnormalities range from scoliosis (usually severe) to spinal dysraphic defects. The umbilical cord is short and adherent to the placental membranes. A single umbilical artery is very commonly present, similar to our case presentation.

Because LBWC is incompatible with life, it is important to diagnose the lesions prenatally and to differentiate them from other anterior abdominal wall defects [7]. LBWC should be differentiated from common abdominal wall defects such as gastroschisis, omphalocele and uncommon entities like ectopia cordis, amniotic band syndrome, cloacal dystrophy and urachal cyst. One of the principal parameters that assist this differentiation is the site. While ectopia cordis is typically located at anterior aspect of thorax, gastroschisis and omphalocele are localized to the umbilical and paraumbilical areas. Similarly, cloacal dystrophy, urachal cyst are entities involving the lower abdominal wall. Other useful parameters that aid in deriving a specific 


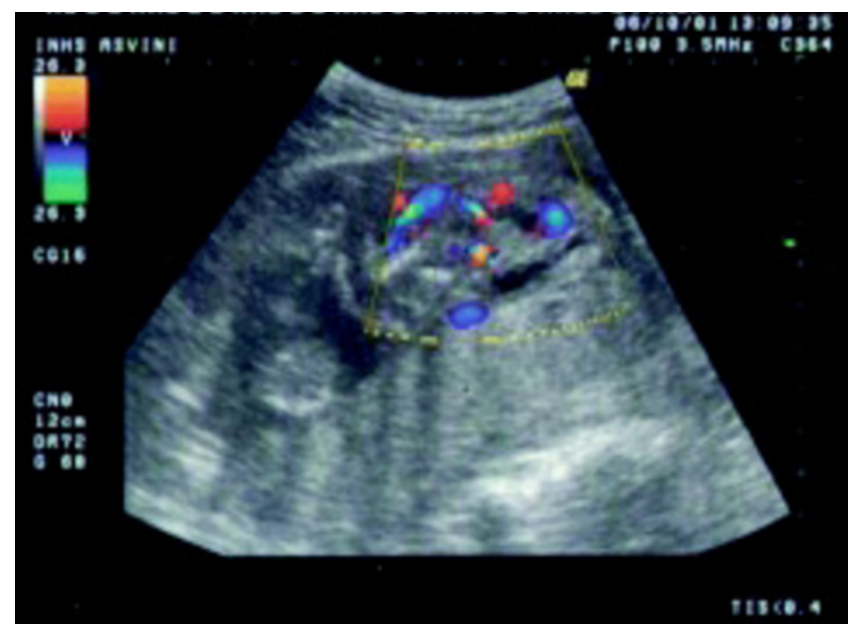

Fig. 1: Colour flow imaging scans of the fetus showing a large ill defined abdominal wall defect through which the abdominal contents herniate into the extra embryonic coelom. The eviscerated organs form a complex, bizarre appearing mass entangled with membranes. The diaphragm is absent

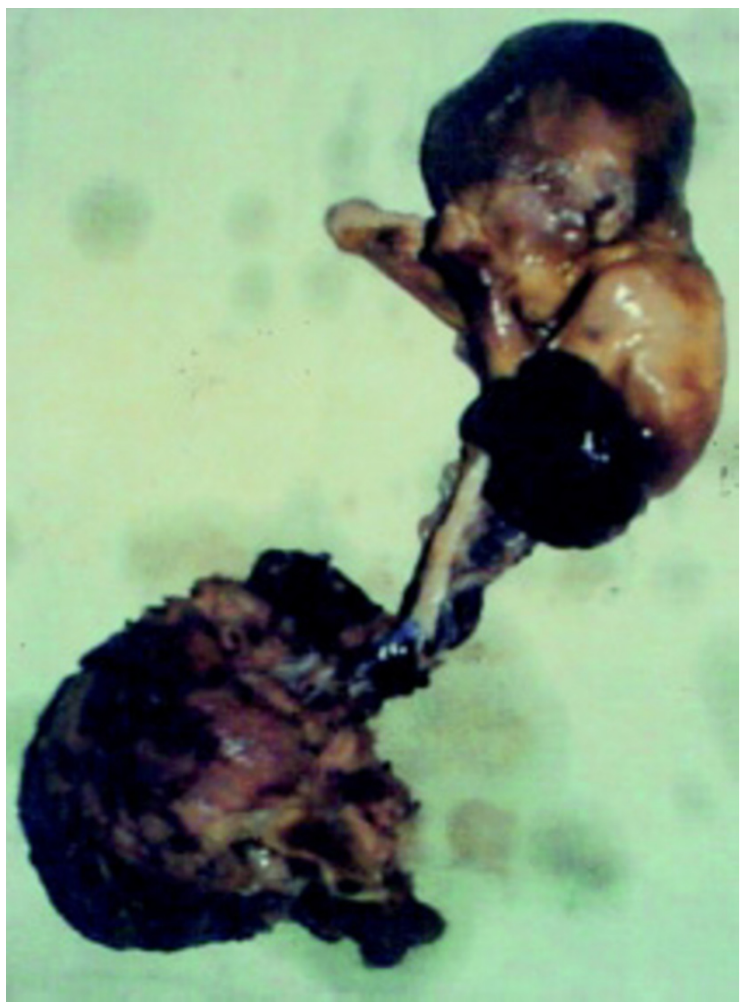

Fig. 3: Photographs of the aborted fetus swhows herniation of abdominal contents through a large defect with the eviscerated organs form a compled bizarre appearing mass entangled with membranes. An extracorporeal liver is present. The umbilical cord is short, straight, incompletely covered by amnion and adherent to the placental membranes as well as the eviscerated mass

diagnosis include the presence of membranes covering, the contents of herniated sac, any associated bowel abnormalities, the presence or absence of urinary bladder, scoliosis and limb defects, the presence or absence of other random defects such as facial cleft. Lastly, a combination of omphalocele and scoliosis should raise

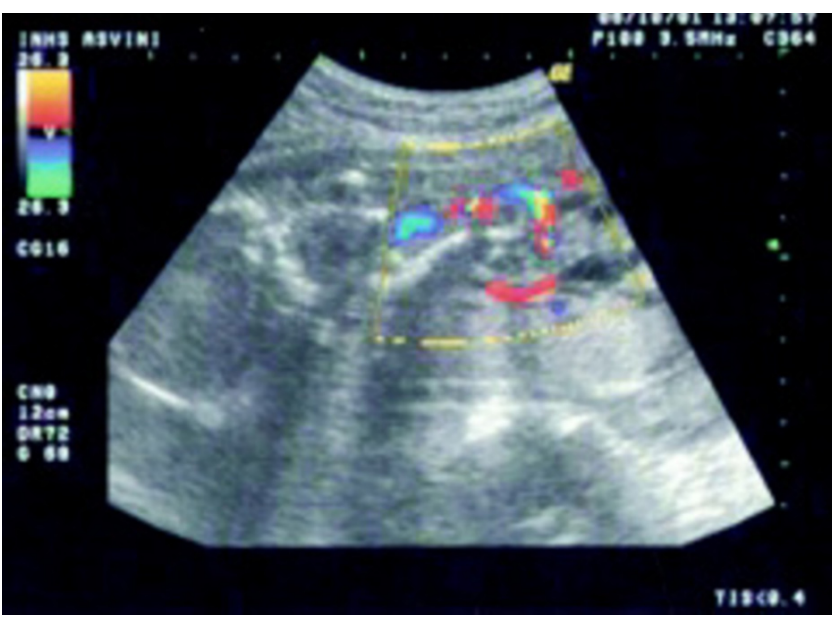

Fig. 2 : Colour flow imaging showing a single umbilical artery within a short umbilical cord connecting the fetus

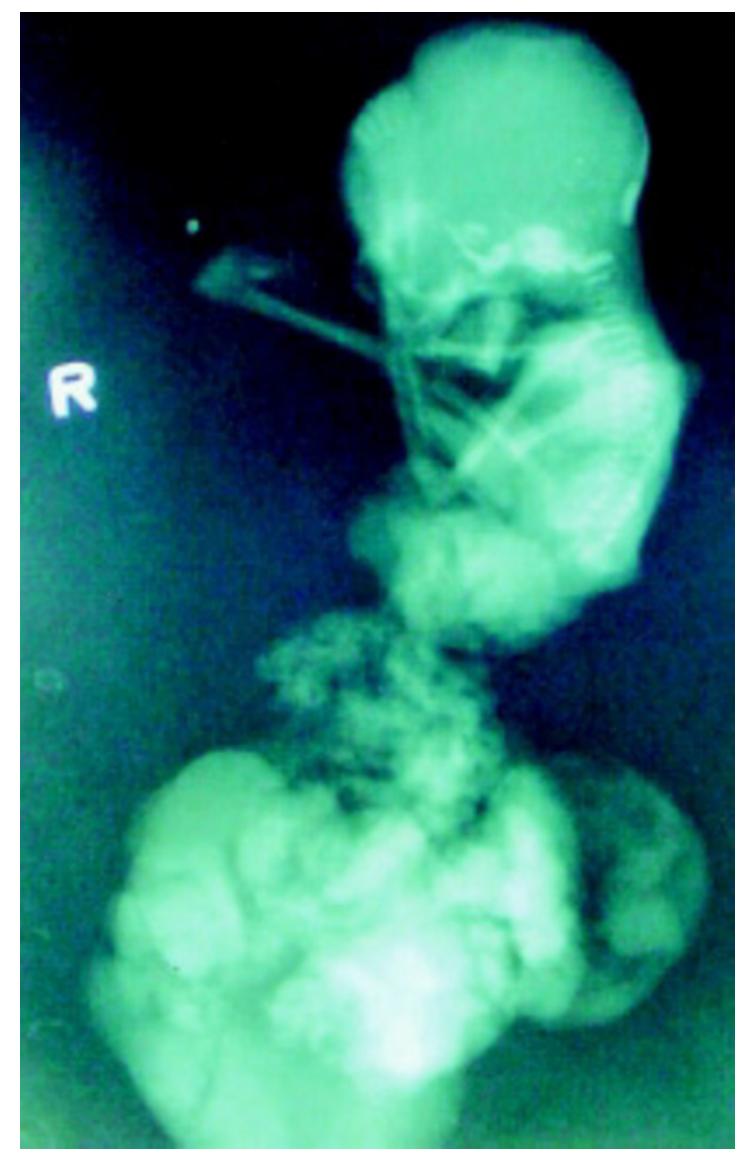

Fig. 4 : Plain radiographs of the aborted fetus display markedly diminished thoracic and abdominal circumference. The eviscerated organs form a compled, bizarre appearing mass. Clubfoot is present at right side

the suspicion of LBWC.

\section{References}

1. Kamudhamas A, Manusook S. Limb-body wall complex, report of 2 cases with their quintessence is prenatal diagnosis. J Med Assoc Thai 2001;84(4):602-8.

2. Patten RM, Van Allen M, Mack LA et al. Limb-body wall complex : in utero sonographic diagnosis of a complicated fetal 
malformation. Am J Roentgenol 1986;146(5):1019-24.

3. Smith DW. Recognizable Patterns of Human Malformation, $3^{\text {rd }}$ ed, Philadelphia, WB Saunders Co, 1981;488-96.

4. Van Allen MI, Curry C, Gallgher. Limb-body-wall complex I : Pathogenesis. Am J Med Genet 1987;28:529-48.

5. Van Allen MI, Curry C, Gallagher. Limb -body-wall complex
II : Limb and spine defects. Am J Med Genet 1987;28:549-65.

6. Deruelle P, Hay R, Subtil D et al. Antenatal diagnosis of limb body wall complex. Gynecol Obstet Biol Reprod (Paris) 2000;29(4):385-91.

7. Russo R, D'Armiento M, Angrisani P, Vecchione R. Limb body wall complex : a critical review and a nosological proposal. Am J Med Genet 1993;1:47(6):893-900.

\section{MEDICAL PHILATELY}

\section{Sir Ronald Ross}

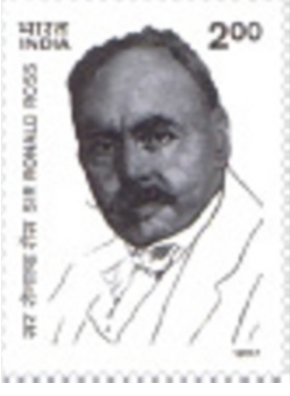

ir R onald R os s was born on May 13, 1857 (a turbulent year in his tory of India) at Almora, a hill s tation in U ttranchal s tate of India. His father, S ir C.C.G R os s was a General in the English Army posted in India. After his schooling in England, he studied medicine at $\mathrm{S} \dagger$ B artholomew's hos pital in London. He joined Indian Medical S ervice in 1881. He chos e to train in bacteriology and to s pecialize in tropical dis eas es .

He commenced the study of malaria in 1892. In 1894, he determined to make experimental inves tigations of the hypothes is of $L$ averan and Mans on that mos quitoes were connected with propagation of malaria. He succeeded after two and a half years. On 20th Augus 11897 at $S$ ecunderabad (Andhra Pradesh in India), R oss made the dis covery of pigmented oocys ts of malarial paras ite in anopheles mos quito (called dapple-winged mos quito by Ross). Thus he solved the biggest puzzle in tropical medicine by demonstrating transmission of malaria by mos quitoes. S ir Mans on announced R os s's monumental result at the E dinburgh meeting of B ritish Medical As s ociation on 28 th July 1898.

In 1901 R oss was elected a fellow of the R oyal College of $S$ urgeons of England and als 0 a fellow of R oyal S ociety, of which he became Vice-P resident from 1911-1913. He was awarded Nobel prize for medicine in 1902. He was knighted in 1911. During his active career Ross's interest lay mainly in initiation of meas ures for prevention of malaria in different countries of the world. $\mathrm{H}$ is interest in mathematics led him to develop mathematical models for s tudy of epidemiology of malaria. He was the first to employ counting for malarial paras ites to as sess the intens ity of malarial infection. His intense involvement with malaria did not prevent him from finding time and mental energy for artis tic purs uits. He was a poet, writer and painter. His poetic works in particular won him wide acclaim. He died after a long illness at R os s Ins titute L ondon on S ep 6, 1932. The illus trated stamp (Rs. 2.00, single colour, perforation $13 \times 13$ ) was is sued by Department of P os ts, India on 20th Aug 1997 to commemorate 100 years of the epoch making dis covery of $S$ ir R onald R oss.

Contributed by :

Col J Dutta* , Dr Abhijeet ${ }^{+}$, Col KP Anand ${ }^{\sharp}$

*Associate Professor, Department of Preventive \& Social Medicine,"Associate Professor, Department of Medicine, Armed Forces Medical College, Pune - 411 040, 'Department of Public Health, Johns Hopkins University. 